\title{
Severe Gangrene Accompanied by Varicella Zoster Virus-Related Vasculitis Mimicking Rheumatoid Vasculitis
}

\author{
Aya Tanaka ${ }^{a, b}$ Nagako Hayaishi ${ }^{b}$ Yukari Kondo ${ }^{b}$ Kishiro Kurachi ${ }^{b}$ \\ Atsushi Tanemura ${ }^{a}$ Ichiro Katayama ${ }^{a}$ \\ ${ }^{a}$ Department of Dermatology, Osaka University Graduate School of Medicine, Suita, and \\ ${ }^{b}$ Department of Dermatology, Toyonaka Municipal Hospital, Toyonaka, Japan
}

\section{Key Words}

Varicella zoster virus $\cdot$ Acral gangrene $\cdot$ Immunosuppression $\cdot$ Rheumatoid arthritis

\begin{abstract}
Herpes zoster infection occurs more frequently and severely in immunosuppressed populations. However, the condition sometimes presents with atypical clinical manifestations of the skin, which makes it difficult to reach a correct diagnosis. We experienced a case of acral gangrene caused by varicella zoster virus (VZV)-related vasculitis in a rheumatoid arthritis (RA) patient. Histologically, necrotic vasculitis was observed; however, there were initially no findings in the epidermis suggestive of a viral infection. We thought that the skin ulcer was related to rheumatoid vasculitis. However, an immunohistochemical analysis for VZV confirmed VZV infection in the vascular endothelium of the dermis, leading to effective treatment with intravenous acyclovir. Since various pathogenic skin phenotypes are observed in RA patients, modified according to the status of immunosuppression, clinicians must recognize the variation in typical and atypical manifestations in order to manage these patients.

(c) 2014 S. Karger AG, Basel
\end{abstract}

\section{Introduction}

Herpes zoster infection is a viral disease caused by the reactivation of varicella zoster virus (VZV) presenting with characteristic painful skin rashes with blisters. The disease 
appears more often in immunosuppressed populations and involves various atypical clinical manifestations of the skin [1, 2], even in patients without epidermal involvement [3, 4].

On the other hand, rheumatoid arthritis (RA) is often accompanied by various skin complications related to granulomatous changes and cutaneous vasculitis [5]. Furthermore, such patients are often under an immunosuppressive state, with a high risk of developing viral, bacterial and fungal infections due to RA treatment [6]. In addition, the increasing incidence of various adverse effects on the skin caused by biologic agents [7] makes it more complicated to diagnose skin lesions in patients with RA.

We herein report a case of severe gangrene related to vasculitis caused by VZV infection in an RA patient, which was initially considered to be due to rheumatoid vasculitis. The lesions dramatically improved following treatment with intravenous acyclovir and healed without surgical intervention.

\section{Case Report}

A 72-year-old male who had been under treatment for RA with methotrexate ( $8 \mathrm{mg} /$ week), prednisolone $(5 \mathrm{mg} /$ day) and bucillamine $(200 \mathrm{mg} /$ day $)$ developed a skin ulcer on the left fifth toe and lymphadenitis on the left inguinal region. Although Streptococcus haemolyticus was detected in the skin ulcer and systemic antibiotics were administered, the skin ulcer expanded in size and depth, exposing the extensor tendon, and another ulcer consistent with scattered necrosis subsequently appeared on the dorsum of the left foot. A microscopic test for superficial mycoses, the Tzanck test and a cultivation test for mycobacterial infection were all negative. Furthermore, subcutaneous nodules also appeared on the left patella. A biopsy specimen obtained from the skin around the ulcer and subcutaneous nodule showed necrotic vasculitis although there were no remarkable changes in the epidermis, and we were concerned that the patient had progressed to rheumatoid vasculitis. Two months later, the ulcer on the fifth toe had further expanded with surrounding small erythematous, bullous lesions, while the other ulcer on the dorsal foot had become deeper, exposing the joint capsule (fig. 1). A skin biopsy of the bullous lesion revealed acantholysis and ballooning degeneration of keratinocytes. Leukocytoclastic vasculitis with fibrinoid degeneration was also seen in the reticular dermis and subcutaneous tissue as it was in the findings from the first biopsy specimen (fig. 2a,b). Immunohistochemical staining using a monoclonal antibody recognizing VZV (Novocastra Laboratories, Newcastle, UK) was positive in keratinocytes inside the vesicles, fibroblasts, inflammatory cells and endothelial cells in the underlying dermis (fig. 2c). VZV was identified on a virological culture of a swab.

We also performed immunohistochemistry for VZV in the first biopsy specimen and confirmed positive staining in the cells in the dermis and subcutaneous tissue, including endothelial cells, with negative staining in keratinocytes (fig. 2d). These results indicated that our patient initially developed VZV vasculitis without the epidermal involvement typical of VZV infection. We considered the possibility of repeated VZV reactivation within several months due to immunosuppression. A decreased serum IgG level was also observed on a serological examination. We stopped the methotrexate therapy and started treatment with intravenous acyclovir. The expansion of the ulcer quickly stopped, and the lesion healed within 3 months without surgical treatment. 
Tanaka et al.: Severe Gangrene Accompanied by Varicella Zoster Virus-Related Vasculitis Mimicking Rheumatoid Vasculitis

\section{Discussion}

The first typical clinical manifestation of herpes zoster infection is edematous erythema. The erythema subsequently becomes vesicular and eventually develops pustules. The lesions usually crust over within 7-10 days; however, ulceration sometimes appears. According to the histological analysis, ballooning degeneration was observed in the erythematous and vesicular stages in the keratinocytes around the intraepidermal vesicles and follicular epithelium. In the pustular and ulcerative stages, degenerated or necrotic changes were present, not only in the epidermis but also in the dermis, including leukocytoclastic vasculitis, necrotic vasculitis and hyalinization of collagen fibers, which led to severe tissue injury, resulting in ulceration [8].

When our patient first came to us, he exhibited atypical manifestations of VZV infection, such as deep skin ulcer development without any bullous lesions or evidence of viral infection in keratinocytes in a histological examination and negativity in the Tzanck test. These clinical manifestations mimicked the symptoms of rheumatoid vasculitis; therefore, we initially misdiagnosed him with malignant RA and considered the need to increase the immunosuppression.

In the previous literature, herpes zoster patients in immunosuppressed conditions did not always progress through erythematous and vesicular stages. They exhibited various clinical manifestations, such as nodules and ulcers, or even lacked epidermal lesions [1, 2]. Erhard et al. [3] reported a case of VZV infection without a vesicular stage in an immunocompromised patient, which resulted in obliterative vasculitis. They suggested that it was a case of direct VZV infection to the dermal vessels from adjacent nerves, bypassing the epidermis, which is usually infected first during VZV reactivation from sensory nerves. This infection route may help to explain the occurrence of lesions without evidence of VZV infection in keratinocytes in our patient. Muraki et al. [8] reported that VZV initially involves the epidermis in the erythematous stage, then it subsequently invades the dermis in the vesicular stage and disappears in the early ulcerative stage. Considering the behavior of VZV, our patient presented after having passed through the erythematous and vesicular stages, and the disappearance of VZV in the early ulcerative stage made it difficult for us to reach the correct diagnosis.

We herein presented a case of atypical and severe skin ulcers caused by VZV vasculitis that mimicked rheumatoid vasculitis. It took us several months to make the correct diagnosis and to provide appropriate treatment. Dermatologic problems in RA patients are complicated, including extra-articular manifestations of the disease, adverse effects related to antirheumatic therapy and various infections due to immunosuppression. Clinicians must recognize the variation in typical and atypical manifestations in order to manage these patients.

\section{References}

1 Tresch S, Trueb RM, Kamarachev J, French LE, Hofbauer GF: Disseminated herpes zoster mimicking rheumatoid vasculitis in a rheumatoid arthritis patient on etanercept. Dermatology 2009;219:347-349.

2 Khera P, Haught JM, McSorley J, English JC 3rd: Atypical presentations of herpesvirus infections in patients with chronic lymphocytic leukemia. J Am Acad Dermatol 2009;60:484-486.

-3 Erhard H, Rünger TM, Kreienkamp M, Müller J, Müller-Hermelink HK, Bröcker EB: Atypical varicella-zoster virus infection in an immunocompromised patient: result of a virus-induced vasculitis. J Am Acad Dermatol 1995;32:908-911.

4 Uhoda I, Piérard-Franchimont C, Piérard GE: Varicella-zoster virus vasculitis: a case of recurrent varicella without epidermal involvement. Dermatology 2000;200:173-175. 
Tanaka et al.: Severe Gangrene Accompanied by Varicella Zoster Virus-Related Vasculitis Mimicking Rheumatoid Vasculitis

5 Sayah A, English JC 3rd: Rheumatoid arthritis: a review of the cutaneous manifestations. J Am Acad Dermatol. 2005;53:191-209.

-6 Hanafusa T, Yamaguchi Y, Kitaba S, Katayama I: Intractable wounds from a herpes simplex infection in an immunosuppressed patient with rheumatoid arthritis. Arch Dermatol 2007;143:1340-1342.

7 Furst DE: Development of TNF inhibitor therapies for the treatment of rheumatoid arthritis. Clin Exp Rheumatol 2010;28:S5-S12.

8 Muraki R, Baba T, Iwasaki T, Sata T, Kurata T: Immunohistochemical study of skin lesions in herpes zoster. Virchows Arch A Pathol Anat Histopathol 1992;420:71-76.

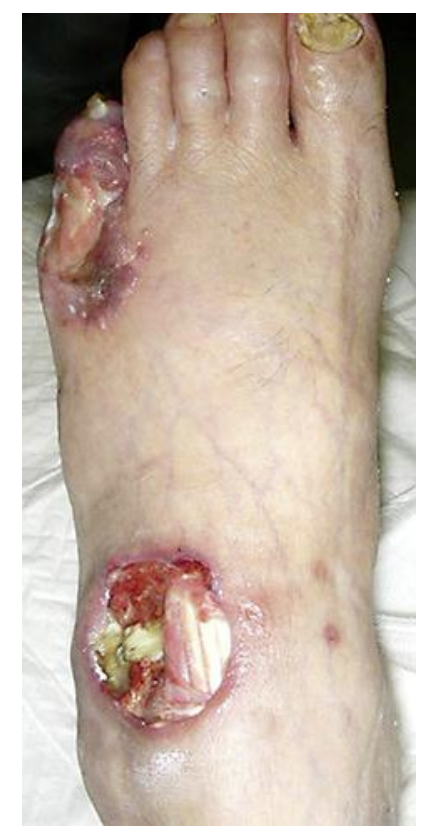

Fig. 1. Clinical appearance of the ulcer 2 months later. The ulcer on the fifth toe had expanded with surrounding small erythematous, bullous lesions, while the ulcer on the dorsum had exposed the joint capsule. 
Tanaka et al.: Severe Gangrene Accompanied by Varicella Zoster Virus-Related Vasculitis Mimicking Rheumatoid Vasculitis
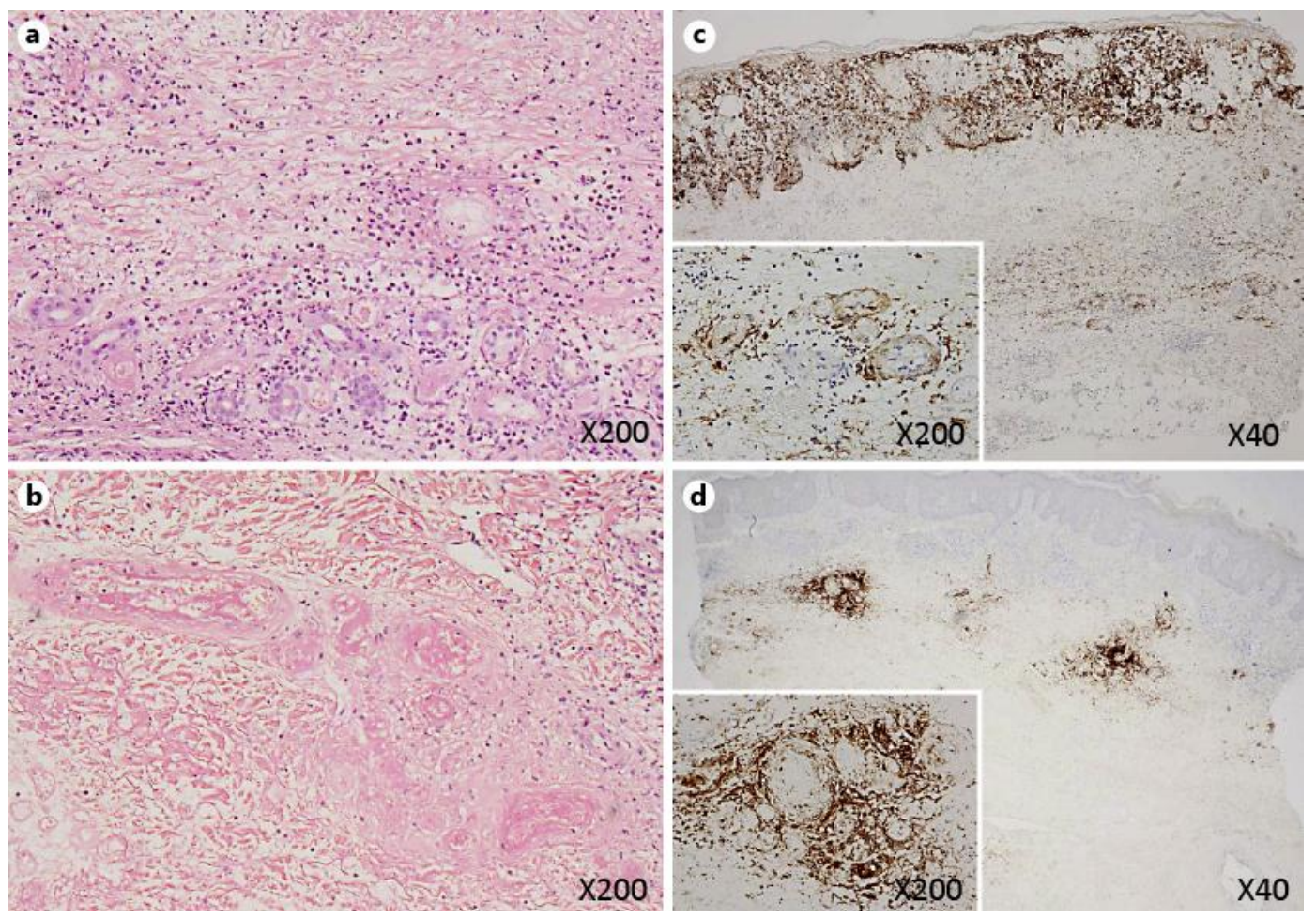

Fig. 2. Hematoxylin- and eosin-stained section of the second biopsy specimen obtained from the bullous lesion showed leukocytoclastic vasculitis with fibrinoid degeneration in the reticular dermis and subcutaneous tissue (a). The first biopsy specimen obtained from the skin around the ulcer on the dorsum also presented perivascular infiltration of inflammatory cells and fibrinoid degeneration as well as necrotic lesions of the vessels in the dermis (b). Immunohistochemistry for VZV in the second biopsy specimen (c) and the first biopsy specimen (d) is shown. Positive staining of endothelial cells and inflammatory cells around the vessels and connective tissue in the dermis and subcutaneous tissue was observed in both specimens. On the other hand, positive staining was noted in keratinocytes only in $\mathbf{c}$ and was negative in $\mathbf{d}$, representing the clinical features of bullous changes. 